\title{
How the Theme of 'Doing and Undoing' Applied to the Action of Exchange Reveals Overlooked Core Ideas in School Mathematics
}

\author{
John Mason 1,2 (D) \\ 1 Department of Mathematics and Statistics, Open University, Milton Keynes MK7 6AA, UK; \\ john.mason@open.ac.uk \\ 2 Department of Education, University of Oxford, 15 Norham Gardens, Oxford OX2 6PY, UK
}

\begin{abstract}
The theme of 'undoing a doing' is applied to the ubiquitous action of exchange, showing how exchange pervades a school's mathematics curriculum. It is possible that many obstacles encountered in school mathematics arise from an impoverished sense of exchange, for learners and possibly for teachers. The approach is phenomenological, in that the reader is urged to undertake the tasks themselves, so that the pedagogical and mathematical comments, and elaborations, may connect directly to immediate experience.
\end{abstract}

Keywords: doing and undoing; exchange; substitution

\section{Introduction}

check for updates

Citation: Mason, J. How the Theme of 'Doing and Undoing' Applied to the Action of Exchange Reveals Overlooked Core Ideas in School Mathematics. Mathematics 2021, 9, 1530. https://doi.org/10.3390/ math9131530

Academic Editor: Michael Voskoglou

Received: 22 May 2021

Accepted: 19 June 2021

Published: 29 June 2021

Publisher's Note: MDPI stays neutral with regard to jurisdictional claims in published maps and institutional affiliations.
Arithmetic is seen here as the study of actions (usually by numbers) on numbers, whereas calculation is an epiphenomenon: useful as a skill, but only to facilitate recognition of relationships between numbers. Particular relationships may, upon articulation and analysis (generalisation), turn out to be properties that hold more generally. This way of thinking sets the scene for the pervasive mathematical theme of inverse, also known as doing and undoing (Gardiner [1,2]; Mason [3]; SMP [4]). Exploiting this theme brings to the surface core ideas, such as exchange, which, although appearing sporadically in most curricula, actually underpins a great deal of school mathematics.

The idea that young children often struggle with the notion of coinage can be well attested by visiting any primary school. In one school that I visited, 'making change with pretend coins' was a topic visited at every grade level in the school, and yet there were children, at every grade level, who failed to come to grips with the dual notion of counting the number of objects and adding the value assigned to each of those objects. Something similar happens with arithmetic using base 10, requiring the exchange of one ten for 10 ones, etc. I conjecture that the notion of exchange lies at the heart of this joint perception, and that incomplete appreciation and comprehension of exchange lies at the heart of the learner's difficulties. The essence of exchange underpins barter, the subject of many medieval 'word problems' (Mason [5]), leading to the use of coins. As it turns out, exchange pervades school mathematics, for example, in dealing with money, fractions, and arithmetic in base 10, as well as substitution in algebra, particularly appearing in function notation, such as $f(2 x+3)$.

Fractions are commonly taught year-after-year because learners do not fully comprehend arithmetic with fractions, much less appreciate its use. Again, the notion of exchange underpins the transition from fractions as actions or operators on numbers, to rational numbers as numbers. Modular arithmetic too, is based on the notion of equivalence classes, which depends on the notion of exchange.

The aim here is to bring to the surface a number of different contexts in which doing and undoing brings to attention significant mathematical relationships, and to justify the assertion that the actions of arithmetic (add, subtract, multiply, divide, exponentiation) are all based on comprehending exchange. 
This paper is not, and does not pretend to be, reporting empirical studies. Rather it is a study of the manifestation of didactic transpositions (Chevallard [6], Bosch and Gascón [7]). Rather than being satisfied to outline some mathematical relationships in the expectation that teaching them is unproblematic, the thrust is to bring to the surface pedagogical and didactic choices, based on delineating core awarenesses, which, in a different discourse, can be seen as teacher-perceived critical or pivotal aspects contributing to appreciation and comprehension (understanding). In contrast to the notion of intended learning trajectory (Simon and Tzur [8]), I take what is called by some a rhizomatic approach (Deleuze and Guattari [9]), in which each learner negotiates a personal path in and through a domain, directed by personal shifts of attention and idiosyncratic associations and experience. Here, each topic is perceived as a field of exploration and study (in the sense of Vergnaud [10]), consisting of senses-of and facility with language and techniques, awarenesses and misapprehensions, and origins and uses (Mason and Johnston-Wilder [11]). The use of 'senses-of' highlights the gestalt experience of being informed by some sense of form, not currently (and perhaps not possibly) analysed in detail.

To put it another way - the aim here is to highlight core awarenesses (critical aspects, pivotal points, threshold concepts) that may be overlooked in manifesting a single-track planned curriculum, and to highlight pedagogic issues and possibilities when introducing to young children the experience of exchange, the undoing of an action, and to indicate ways in which these themes both pervade all levels of school mathematics.

\section{Themes, Actions and Awarenesses}

The core stance taken here is that any effective didactic contract (Brousseau [12]) calls upon learners to undertake tasks thoughtfully and mathematically: the aim is not to 'get answers' (which can often be achieved simply by copying), but to experience mathematical thinking (Mason, Burton, and Stacey [13]; Cuoco, Goldenberg, and Mark [14]; Gardiner [1]; Schoenfeld [15]). The specific theme highlighted here is the theme of doing and undoing (Mason [16,17]; Gardiner [2]): whenever an action or sequence of actions can be used to get an answer (a doing), it is useful to consider the undoing, the action which starts from the result, and leads to the input data (Mason and Johnston-Wilder [11,18]). Indeed, arithmetic, seen as actions (of numbers) on numbers, is largely the study of doing and undoing.

The term awareness is used here in the sense of Gattegno ([19]), referring to the internalisation of conditions (which one may be explicitly, or only vaguely aware of, and which often lie below the level of consciousness altogether). Those 'conditions' are associated with actions, which then become available to be enacted in the future. Awareness encompasses both the sensitivities to notice and associated actions. Gattegno used to say that "only awareness is educable", referring to the internalisation of actions and sensitivities to make those actions become available for enaction, in order, it seems to me, to distinguish between the development of awareness and mere training of behaviour (hence, "only behaviour is trainable" (Mason [20,21]). Of course, neither is sufficient on its own, because an internalised action requires fluency and facility in order to be useful, calling upon as little attention as possible in order to be enacted correctly. This leaves room for attending to larger, more encompassing goals, and to watch out for wrinkles or obstacles during the enactment. It is worth mentioning that what enables training of behaviour and educating of (one's own) awareness is the harnessing of emotion, the source of energy. Hence, "only emotion is harnessable".

The term didactic transposition was coined by Yves Chevallard ([6]) to refer to the transformation (transposition) that takes place when expert awareness is converted into instruction in behaviour (tasks, exposition, and exercises). A similar transposition takes place at many levels of institutionalisation (Bosch and Gascón [7]) as curriculum is gradually turned into a sequence of pedagogic actions initiated by a teacher or author. My concern here is with what tasks the authors intended learners to experience. Such a transformation is particularly noticeable between an expert becoming aware of some mathematical property, perhaps as instantiated in some particular setting, and the construction of tasks 
for the learner, with the aim of prompting learners to have a similar experience. The experiences are necessarily different, because the expert is realising a connection from a basis of experience, whereas the learner is encountering at least some of the ideas, their instantiation, or their connections, ab initio. Consequently, the design of the task sequence, and the invoking of suitable pedagogic or didactic actions, is crucial to overcoming possible chasms arising from effects of the didactic transposition.

Since expert awareness involves a developed sensitivity to certain kinds of details and relationships with access to properties, which may be instantiated, the focus and structure of attention, both of the teacher and the learner, is critical. Where these are misaligned, communication is likely to be, at best, partial, if not impoverished. Because attention is at the core of the teaching-learning process, it is important to become aware, not only of what is being attended to by myself and by learners, but also the form of that attention (Mason [22,23]). It is convenient, and to me helpful, to distinguish between holding wholes (gazing), discerning details, recognising relationships, and perceiving properties as being instantiated, so that reasoning on the basis of agreed properties can be used to justify conjectures, to oneself, to a friend, and then to a sceptic (Mason Burton and Stacey [13]).

\section{Method}

The method undertaken in this paper is phenomenological: the focus is on the potential lived experience of learners, informed by my own awareness of my own lived experience. Consequently, the exposition is centred on a sequence of tasks in each of several domains, developed from my own experiences. Some of the tasks here can be modified for use with very young children; later tasks are aimed at upper primary, then secondary. Task domains constitute aspects of mathematical themes, which I conjecture are often overlooked within school mathematics, to the detriment and impoverishment of learner experience and indeed of learner motivation. Within each domain, sample tasks are proffered with comments about possible pedagogic actions that may be helpful. Answers are rarely if ever given for tasks, because answers are much less important than experiencing various mathematical actions and themes. In the sense of the didactic transposition, the transformation from expert-noticing of themes and actions to learner-noticing and internalising depends on the learner being immersed in similar tasks for a period of time, together with the initiation of pedagogic actions intended to promote awareness, including both invitations to construct one's own examples (Watson and Mason [24]), and one's own personal narrative (Bruner [25]; Chi and Bassok [26]).

In order to participate fully in this 'method' of analysis, it is essential to both read the tasks and engage with them. That means not simply thinking about them, but actually making a record of the work, as well as recording what is noticed in the way of movements of attention, shifts in how you are attending, and the ease or difficulty you experience in attempting to articulate your own personal narrative regarding what the tasks are about for you at that moment. All tasks are designed for this paper, and most will need modification or alternatives in order to be useful for particular learners.

\section{Exchange}

In the Phaedrus dialogue, Plato praised the Egyptians for using objects to teach citizens arithmetic (Hamilton and Cairns [27]). Although the use of counters and other counterlike objects has become common as a context for counting (see for example Mill [28]; Montessori [29]; NRich website [30]; BBC website [31]; Gripton and Pawluch [32]), there are hidden potentials not always exploited, which provide an introduction to a core mathematical idea that also pervades the whole of mathematics: things are things in their own right, but can also be assigned values in relation to each other. Although the tasks that follow may seem elementary at first, it is much more insightful to carry them out physically, to become aware of shifts in attention and core awarenesses and, hence, to get a taste for what learners might experience. 
Throughout these tasks, there are several dimensions of possible variation (Watson and Mason [24]): aspects, which can be varied so that learners appreciate the richness of the situation and so that the intended underlying concept is available to be learned, as indicated by the principle of variation (Marton and Booth [33]; Marton [34]). For example, the colours may of course be altered, with care not to run into confusion for colour-blind children; the cardinalities, and the exchange ratios can also all be varied. Multiple exposure to variations is required only until learners give evidence that they appreciate these dimensions. Each dimension of a possible variation has a corresponding range of permissible change, within which the phenomenon or concept is preserved.

\subsection{Early-Exchange Tasks}

The first two tasks simply draw attention to acts of counting, which learners are here assumed to be familiar and competent with. The tasks also introduce the mathematical theme of doing and undoing.

\footnotetext{
Task 1: supplementing

Start with a pile of red counters. Now supplement this with some blue counters. What is the relationship between the number of red counters, the number of blue counters, and the final number of both sets of counters altogether?
}

Tasks like this appear in the first years of formal schooling in most countries. Notice that the two original piles are exchanged for a single conglomerate pile. Although apparently elementary, learners need to discern details (in this case, colours, but perhaps not size or thickness), and then to undertake the act of counting, which itself requires discerning entities as things yet to be counted (as distinct from already counted things, other nearby objects, or counters of a different size, shape, or colour). This implies a sense of relationship, which must be held in mind: objects and the qualities of having-been-counted, not-to-be-counted, or not-as-yet-uncounted. Behind such tasks is the eventual awareness that the number of red counters and the number of blue counters together can be obtained through counting-all or counting-on, in order to reach the total number of counters. Notice that in each case, the number is 'a number of some things': it is like an action on or a quality assigned dynamically to those things. In Maori (Barton, Fairhall, and Trinick, [35]), and in Mi'kmaq (Borden [36]), numbers are based on verbs, as an action performed by a person, which avoids the question of "what is three about this item?" when counting three objects.

A further object of learning (Marton [34]) is the action of counting-on, and efficient use of this action (start with the larger number). Counting-on efficiently is notoriously difficult to 'teach'. It seems to require the children to come to the realisation for themselves-that in order to count a compound set of objects, when some have already been counted, it is sufficient to start as if the initial count had already been performed, and then to realise that to add 9 to 3 is the same as adding 3 to 9 , so you can count on from 9 rather than from 3 . What is sometimes overlooked is that counting-on has an inverse action: more of this later!

Task 2: undoing supplementing

Start with a pile of red and blue counters. Ask participants to separate into two piles, one all-red and the other all-blue, and to talk about the relationships between these two numbers and the number of counters altogether.

It is well known that young children need time and experience to be able to articulate relations involving three numbers. It seems to take some time for some children to hold three distinct numbers in mind and to use all three of them in a single utterance. At first, they will say things like "there are 4 more", then "7 is 4 more", and "7 is more than 3", before eventually assembling "7 is 4 more than 3 " (James and Mason [37]). Three numbers each have to be discerned as cardinalities, held in mind, and related. It is a non-trivial shift then to see the specific relationship as an instance of the general property that ' 3 of anything together with 4 of anything else is 7 things all together'. 
At some point, it is useful to direct the learner's attention to the relationship between the two tasks. They may be so immersed in the action that they are unaware of the task as a task, as something that initiates activity; they may be aware of the task as calling upon an action, but not be aware of that action as something they can initiate for themselves in other circumstances. To perceive the tasks as related, must first discern them as different, before recognising a relation between them. Over time, this awareness can be associated with the action of formulating and undertaking the undoing of counting tasks like this one, and then more generally.

There are of course many different tasks, which can be used to promote the shifting of attention from simply counting, to adding and subtracting, which pervade the literature on early number. The didactic transposition can alert teachers to constructing tasks that put children in situations that call upon them to stretch or extend their awareness of what they are doing, and how they are doing it, rather than tasks which consist of a sequence of 'doings' without reflection and persona narratives. In other words, teachers can be alerted to seeking tasks and associated pedagogic actions, which bring learners into contact with pivotal or core awarenesses.

\subsection{Exchange as First Encounter with Arithmetic beyond Adding and Subtracting}

The notion of exchanging gifts, which develops into a more complex activity of bartering, is something that most children experience very early. It may be at an abstract level (behave this way and you will be rewarded), or allowing another child to play with your toys so that you can have the same privilege either now or later, and it is observed at a practical level when adults pay for things (when using cash ... an increasingly rare phenomenon!). Exchange depends on two simultaneous perspectives: the thing itself and its perceived or assigned value in relation to other things. It is a reasonable conjecture that when young children make mistakes with coins, it is because their attention alights on counting rather than on value.

Task 3: elementary exchange

Make a pile of red counters.

Now exchange each red counter for 3 blue counters.

At first, the tendency is to form a new pile of blue counters. If no one thinks to lay out the exchange in a visually transparent form, participants can be asked how they know that it was done correctly, and be asked to arrange the counters so that they and others can see directly that the exchange has been done correctly. Indirect pedagogic actions ("How do you know you have done the exchange correctly?" rather than direct prompts ("Please display the counters so that I can see immediately that the exchange has been done correctly") are more likely to call upon the learner to make choices and, so, experience the action more fully for themselves.

Children can be asked to make a written record of the exchange, and then perhaps exchange records and invited to re-enact someone else's record. Note the opportunity to work with diagrams as records, and then symbols, with movement between the two.

Task 4: undoing an exchange

Use the layout chosen in task 3 to see how collections of 3 blue counters could each be exchanged for a single red counter.

For children who can already count, questions can be asked about how many red, how many blue, and articulate the relation between red and blue, to be introduced as deemed appropriate. Note that, starting with a pile of blues, this undoing action may result in some leftover blue counters (remainders) which must be included in any written record. Notice that the red counters are 'counting' the number of blue-triples.

Having done a few tasks like this, children already familiar with arithmetical operations of multiplication and addition can then be asked: 
Task 5: arithmetic meaning

What arithmetical action corresponds to the action in task 3 ?

What arithmetical action corresponds to the action in task 4 ?

This is the critical pedagogic move from specific to general, from doing to construing, with each learner forming their own personal narrative for what they were doing. If noone thinks to suggest changing the numbers in the exchange, then participants can be asked what could be changed, and still, the task calls upon the same actions. The idea is to bring to the learner's attention the possible variations of which the teacher is aware, and which comprise the desired object of learning, the core awareness (action and trigger). For example:

Task 6: more complex exchange

Make a pile of red counters.

Exchange 2 red counters for 3 blue counters as often as possible.

Rehearse the form of task exchange with the new situation.

Task 7: compound exchange

First, exchange 2 red counters for 3 blue counters as often as possible. Then exchange 2 blue counters for 1 green counter as often as possible.

What is the same and what is different about this exchange, and directly exchanging 4 red counters for 3 green counters?

Note that starting with 3 red counters will give (slightly) different results for the two exchanges, depending on the starting numbers ... why? Remainders are another stumbling block for learners, especially when they are converted into fractions of the original divisor, as in decimals. It makes sense therefore to provide enough experience and opportunity to talk about their actions that learners internalise awareness of what remainders are, how they arise, and what they mean.

\subsection{Commentary}

The variations from task-to-task could be seen as discerning didactic variables, or use of the principle of variation.

Although these tasks are cast in terms of counting, and aimed at young children, they also provide background experience of ratio, especially if juxtaposed with an experience of 'berry-picking': repeatedly enacting "one for me and three for the pot"; "one for me and three for the pot" ${ }^{\prime \prime}$. .

The notion of exchange is rudimentary, as evidenced by bartering as the earliest form of trade. It provides opportunity to get a sense-of multiplication and division before these are talked about, as well as a sense-of objects having value in relationship, as well as numerosity. Having a developed sense-of provides access to a gestalt form that literally informs actions. Just as 'numeral as adjective 'becomes 'number as noun', so 'having a value in relationship' becomes 'having a value'.

\section{Four Operations}

Arithmetic is usually introduced as the study of binary operations, such as $3+4=7$ and $3 \times 4=12$. It has often been pointed out that the ' $=$ ' sign is frequently identified with the 'do it' button on a calculator, whereas in mathematics it refers to different names for the same thing, perhaps locally, perhaps globally. Thus, $3+4$ and 7 are different names for the same thing. Writing $7=3+4$ encourages access to the action of decomposing, whereas $3+4=7$ promotes access to the action of composing or combining. The important thing is that each can be exchanged for the other whenever it is helpful, and indeed for other combinations such as $1+2+4$, and $5+2$. Furthermore, there is considerable advantage to thinking of actions not as binary operations, but as unary operations: in this way, numbers can be thought of as operators on single numbers, as follows: 
Task 8: additive actions

What arithmetic action undoes 'the action of adding $3^{\prime}$ ?

What arithmetic action undoes 'the action of subtracting $4^{\prime}$ ?

What single arithmetic action has the same effect as the combination of first 'adding $3^{\prime}$, then

'subtracting $4^{\prime}$ ?

What is the same and what is different about the compound action 'adding $3^{\prime}$ then 'subtracting $4^{\prime}$, and the compound action 'subtracting $4^{\prime}$ then 'adding $3^{\prime}$ ?

Notice the generality underlying the task statement. Core awarenesses include the notion of inverse or undoing, and that to undo a sequence of actions often requires that they be undone in the reverse order. An opportunity for discussion arises when children consider which pairs of actions on material objects have to be undone in the reverse order and for which does it not matter, and for which is there no possibility of 'undoing'?

Through awareness of these key ideas, as general principles, teachers can immerse learners in useful variation in which one aspect is varied while others are held invariant, to make the varied aspect available for encountering, recognising, and learning. For example, varying only the ' 3 ', then only the ' 4 ' can make learning much more efficient than varying both simultaneously, because learners can experience and acknowledge the varied dimension more efficiently than when two independent dimensions are varied, seemingly at random.

Task 9: multiplicative actions

What action undoes 'multiply by $3^{\prime}$ ? What action undoes 'divide by $4^{\prime}$ ?

What is the same and what is different about the compound action 'multiply by $3^{\prime}$ then 'divide by $4^{\prime}$ and the compound action 'divide by $4^{\prime}$ then 'multiply by $3^{\prime}$ ?

Enough tasks like these (which aspects could be varied in a structured manner?) need to be experienced so that the doing-undoing relationship is deeply embedded.

The next task then exploits this sense-of.

Task 10: two interpretations

What single action undoes the action of 'multiply by three-quarters'?

What compound action undoes the compound action of 'multiply by 3 then divide by 4 '?

One of the most frequently asked questions in professional development sessions for primary teachers is 'why does flip and multiply' work for dividing by fractions? The previous task not only provides an answer, but also reveals a way of thinking throughout mathematics, exploiting the pervasive theme of 'doing and undoing'. Having multiple ways of describing an action (divide by $\frac{3}{4}$; divide by 3 and multiply by 4 ) affords richness in appreciation as well as comprehension. Notice that multiply by three-quarters can be exchanged for either of the compound actions 'multiply by 3 then divide by 4 ', and 'divide by 4 then multiply by $3^{\prime}$.

\subsection{Exploring 'Subtracting From'}

The action of counting-on turns out to have an associated inverse or undoing.

Task 11: subtracting from

What action undoes the action of 'subtract from 7 '?

Seeking to undo the action 'subtracting from 7 ' often catches people. In my experience, people seem to lose sight of what it is they are trying to find. Suppose someone announces the result of subtracting some (perhaps favourite) number from 7 . How could you recover their number? Sometimes people offer "add to 7", which, if interpreted as "what must you add to your number to make the sum up to 7" is precisely what "subtract from 7" means, and is the inverse of counting-on, because it is "what must you count-on by in order to get to 7 ?"! It can take several minutes to get this clear in your head, however. Of course, ' 7 ' is a placeholder and any other number (rational, negative, real ... ) could be used in its stead, depending on the mathematical sophistication of the learners. 
There is plenty to explore about the "subtract from" actions, including the fact that not only are they self-inverse, but composing any odd number of them has the same effect as a single "subtract from", (their own undoing), while composing any even number of them has the same effect as a single "add on" action. Furthermore, certain (other) binary operations also have an associated from action, which is self-inverse. For example, the binary operation

$a \circ b=a b+a+b$ is associated with the unary operation $\beta_{b}(a)=a \circ b=a b+a+b$ which has an associated from action $\hat{\beta}_{a}(x)=\frac{x-a}{a+1}$ as long as $a \neq-1$.

\subsection{Arithmetic as One Dimension; Geometry as Two or More Dimensions}

Ordinary arithmetic can be thought of as geometry in one dimension. 'Subtract from $a^{\prime}$ on a number line can be seen geometrically as a translation of $a$ by $-x$ or as a reflection of $x$ in the point $a / 2$. A two-dimensional analogue of 'subtract from' is reflection in a point. Reflections are self-inverse transformations. The combination of three reflections in points $A$ then $B$ then $C$ is also self-inverse, as is any odd number of point reflections. Self-inverse actions, known to mathematicians as involutions, play a key role in comprehending the structure of any set of actions. This applies both in arithmetic and in geometry (Thomsen [38]).

Notice the potency of exchanges, which are self-inverse, and the exchange of a composition of actions with a single action (paralleling the case in arithmetic).

\subsection{Complementarity}

There is considerable overlap between the notion of undoing an action and complementarity, whenever it is possible to go back and forth between the actions. For example, young children are often urged to internalise complements-to-ten because it is useful when performing multi-digit subtraction. However, complementarity appears in many other guises. For example, complements-to- 9 .

\footnotetext{
Task 12: converting subtraction into addition

What action undoes 'form the nine's complements of each digit' of a numeral?

Why do the following procedures always work?

To subtract one multi-digit number $B$ from another, $A$, form the number consisting of the $9^{\prime} \mathrm{s}$ complement of the digits of $B$ (first inserting leading $0 \mathrm{~s}$ in $B$ to match the number of digits in $A$ ). Add this to the number $A$. Now delete the leading 1-digit of the answer, and add 1 to that answer. To subtract one multi-digit number $B$ from another, $A$, form the nines' complement of $A$; add to $B$ and form the nines' complement of the result.
}

Here the object of learning switches from doing subtraction to trying to see how and why some action works. It might, in passing, provide an easier algorithm than the usual one, which involves complements to different teen numbers, and keeping track of the tens digit.

Pedagogically, there are, as always, multiple choices. The teacher could perform a few examples and then ask learners to articulate what they saw being done, before then trying to explain why it works, or they could articulate the sequence of actions. To make the method memorable, it is likely that learners will have to come to the realisation of why it works, for themselves, rather than being shown. Might one start with 3-digit numbers, or work up through 1 and 2 digit numbers to multi-digit numbers?

\section{Equivalence as Permissible Exchange}

That learners struggle with the arithmetic of fractions is evident to anyone teaching upper primary and lower secondary. Again, my conjecture is that this struggle is due to insufficient familiarity with, and focus of attention on, exchange. As indicated above, there are plenty of opportunities to exploit exchange of names, such as $3+4$ and 7 , and exchange of actions, so that exchange not only becomes familiar, but is also integrated into available actions. 
A fraction is most usefully seen as an operation on objects, including numbers. The reason is that with fractions it is vital to keep in touch with what the whole is. Thus, comparing $1 / 2$ and $2 / 3$ makes no sense unless they are seen as fractions of the same whole, as operators on a common object. One observes however that acting $2 / 4$ on things and $3 / 6$ on the same things, always gives the same result. Exchanging the operator $2 / 4$ and $3 / 6$ in a calculation makes no difference to the result. In mathematical terms, these are of equivalent value, in that as actions, they always give the same (equivalent) result. In the same way, any two coins or notes of the same denomination can be used interchangeably, just as two same-sized plates or two same-sized bowls can be used interchangeably in the kitchen.

Task 13: Equivalent Exchanges?

Form two piles of red counters with the same number in each.

For one, repeatedly exchange 2 reds for 4 blues, while for the second pile, repeated exchange 3 reds for 6 blues. Will the results be exactly the same? What fraction of the blue piles are the original red piles?

As long as the number of reds is divisible by both 2 and 3, the number of blues in the two piles will be the same, and there will be no remainders. For other numbers of reds, repeatedly exchanging two reds for four blues in one pile, and repeatedly exchanging three reds for six blues in the other pile, will not always appear to give the same result: for example, with 9 reds, there will be 16 blues and 1 red left over after the first exchange, and in the other case there will be 18 blues. It is because, in both cases, 1 red is effectively being exchanged for 2 blues, so 16 blues and 1 red is equivalent to, or has the same value as, 18 blues. Exchanging 2 reds for 4 blues can be built from repeatedly exchanging 1 red for 2 blues, and the same goes for exchanging 3 reds for 6 blues.

Equivalence of fractions, forming rational numbers, can also be used to explain division by rationals.

To divide $\frac{a}{b}$ by $\frac{c}{d}$ first exchange $\frac{a}{b}$ for $\frac{a c}{b c}$ which is now divisible by $c$ to give $\frac{a}{b c}$. To divide by $\frac{1}{d}$ is to multiply by $d$, so the result is $\frac{a d}{b c}$. $\left(\frac{a}{b}\right)\left(\frac{d}{c}\right)$.

Or again, exchange $\frac{a}{b}$ for $\frac{a d}{b d}$ and $\frac{c}{d}$ for $\frac{b c}{b d}$. Then $\frac{a}{b} \div \frac{c}{d}=\frac{a d}{b d} \div \frac{b c}{b d}=a d \div b c=\frac{a d}{b c}=$

Note that even with efficient use of counting-on, exchanging $9+3$ for $3+9$ is exchanging one thing for another, which is known to be equivalent.

\section{Modular Arithmetic}

Modular (or clock) arithmetic is again based on equivalence, so working modulo 12 on a clock face, 13 can be exchanged for 1, and so on, as they have the same positions of the hour hand. Equivalence relations are the core of modern mathematics, based on the permissibility of exchanging 'equivalent objects' without affecting calculations or conclusions. Appreciation and comprehension depends on a firm foundation of exchange.

\section{More Advanced Arithmetic}

The role of the tasks in this section is to indicate the plethora of actions involving numbers in school mathematics, which can be cast as doing, and linked with their undoing via the notion of exchange. The same applies to most routine exercises, including wordproblems. With growing experience of this mathematical theme, learners can be encouraged to initiate exploration of undoing for themselves, giving them a greater sense of agency, commitment, and motivation to continue.

\footnotetext{
Task 14: powers and roots

What action undoes the action of 'multiplying 4 copies of some number together'?

What action undoes the action of 'taking the 5th root'?

Is there an action related to powers and roots, which is self-inverse, in the way that subtract from and divide-into are self-inverse?
} 
It turns out that, in order for a binary operation to lead to a self-inverse 'from' action, it has to be both commutative and associative.

Task 15: gcd and lcm

Finding the $\mathrm{lcm}$ (lowest common multiple) of two numbers can be seen as a 'doing'. The associated 'undoing' is: given a number $n$, for how many different pairs of numbers $a$ and $b$ is $n$ the $l \mathrm{~cm}$ of $a$ and $b$ ?

What happens when undoing the $g c d$ (greatest common divisor; highest common factor) of two numbers is considered?

Notice that there are infinitely many pairs of numbers $a$ and $b$ for which $n$ is the $g c d$. Getting learners to extend their sense of number to include the multiplicative structure as well as the additive often requires a significant shift in how they attend to or think about number. The $g c d$ and $l c m$ task requires a shift into multiplicative thinking. The notions of $g c d$ and $l c m$ can be extended to rational numbers, which brings to the fore relationships between $\mathrm{gcd}$ and $\mathrm{lcm}$.

\subsection{Fractional Parts and Percentages}

Given a certain percentage increase, what subsequent percentage decrease returns the original value? For example, I once had to help a salesman who wanted to know what percentage he should add to the price he wanted to receive from me, so that when he applied the required tax percentage, he would get back to the actual price. He had noticed that using the tax percentage itself did not work.

While working with some teachers in the Palouse area of Idaho, it occurred to me to exploit the issue of fractional increase and decrease, in what came to be called the Palouse Relationship.

Task 16: Fractional increase and decrease
\begin{tabular}{|c|l|l|}
\hline$\left(1+\frac{1}{2}\right)\left(1-\frac{1}{3}\right)=\left(1-\frac{1}{3}\right)\left(1+\frac{1}{2}\right)=?$ & $\left(1+\frac{1}{3}\right)\left(1-\frac{1}{4}\right)=\left(1-\frac{1}{4}\right)\left(1+\frac{1}{3}\right)=?$ & $\left(1+\frac{1}{5}\right)\left(1-\frac{1}{6}\right)=\left(1-\frac{1}{6}\right)\left(1+\frac{1}{5}\right)=?$ \\
\hline$\left(1+\frac{2}{3}\right)\left(1-\frac{2}{5}\right)=\left(1-\frac{2}{5}\right)\left(1+\frac{2}{3}\right)=?$ & $\left(1+\frac{3}{7}\right)\left(1-\frac{3}{10}\right)=\left(1-\frac{3}{10}\right)\left(1+\frac{3}{7}\right)=?$ & $\left(1+\frac{5}{9}\right)\left(1-\frac{5}{14}\right)=\left(1-\frac{5}{14}\right)\left(1+\frac{5}{9}\right)=?$ \\
\hline
\end{tabular}

In the table above, possibly using more examples, which you construct for yourself, for each row, express a general relationship of which the three equations are specific instances.

What does each expression say about how to undo a fractional increase or a fractional decrease? How could that be connected to percentage increase and percentage decrease?

The two factors are intended to be perceived of as inverses or undoing's. The three examples in each row have been deliberately chosen not to be 'sequential' in some sense, to encourage the use of the third example as a check of an initial conjecture.

Making a connection between fractional increase and percentage increase may not occur to learners, and so may need to be prompted.

Notice how the theme of doing and undoing is integrated into a task about imagining and expressing generality. Notice also the exchange implied by converting fractions to percentages via denominators of 100 . One contributory factor to learners separating the domain of percentages from the domain of fractions is the inconsistent use of fraction to mean rational, and fraction to mean an action on something. A fraction-of some whole and a percentage-of some whole, both actions, make the exchange of equivalent fractions more transparent. 
7.2. Extending the Palouse Relationship

Task 17: preserving products

\begin{tabular}{|c|c|c|}
\hline$\left(11+\frac{1}{5}\right)\left(7-\frac{1}{8}\right)=\left(7+\frac{1}{3}\right)\left(11-\frac{1}{2}\right)=?$ & $\left(11+\frac{1}{12}\right)\left(7-\frac{1}{19}\right)=\left(7+\frac{1}{14}\right)\left(11-\frac{1}{9}\right)=?$ & $\left(11+\frac{1}{19}\right)\left(7-\frac{1}{18}\right)=\left(7+\frac{1}{25}\right)\left(11-\frac{1}{16}\right)=?$ \\
\hline$\left(33+\frac{3}{5}\right)\left(21-\frac{3}{8}\right)=\left(21+\frac{3}{3}\right)\left(33-\frac{3}{2}\right)=?$ & $\left(33+\frac{3}{12}\right)\left(21-\frac{3}{19}\right)=\left(21+\frac{3}{14}\right)\left(33-\frac{3}{9}\right)=?$ & $\left(33+\frac{3}{19}\right)\left(21-\frac{3}{31}\right)=\left(21+\frac{3}{25}\right)\left(33-\frac{3}{16}\right)=?$ \\
\hline
\end{tabular}

Extend the rows of the table; generalise!

Notice that the core idea is to preserve a product by finding appropriate fractional increased and decreases. This is the pervasive mathematical theme of invariance in the midst of change: what can be changed, and still, some property remains invariant?

The learner is expected to take the first row as a signal to generalise the Palouse relationship to the product $a b$, which involves the Bézout relationship, itself an offshoot of careful book-keeping when using the Euclidean algorithm on $a$ and $b$, in order to solve $b y$ $-a x=1$, and its companion, $a x-b y=1$. Once one solution has been found, subsequent solutions can be found by adjusting the denominators of the fractions. The second row is intended to signal what happens when $a$ and $b$ have a common factor. In both cases, three instances are considered sufficient to suggest a generality involving an invariant (the product), and what is permitted to change while preserving that invariant.

Pedagogically, learners are invited to try to generalise, and this may mean constructing examples for themselves until they can become aware of, and articulate, relationships.

The task provides plenty of opportunity to discern details, to recognise possible relationships, which might then be instances of properties, which can then be used to reason, for example, in justifying a conjectured generality. Underlying the result are pairs of doing-undoing actions, and the sense that the identity can be exchanged with these paired products in situations in which it might be helpful.

\section{Algebra as an Exchange and as a Notation}

This section aims to make the case that algebra, the naming of expressions, depends upon the notion of exchange, and is likely to be easier to grasp if exchange has been integrated into a learner's functioning.

\subsection{Exchange Underpins Algebraic Notation}

What is algebra? Most textbooks, since the 15th century, when algebra began to be widespread, have treated algebra as 'arithmetic with letters'. It is no wonder that so many adults report losing the plot when they first met algebra. Yet, as Dave Hewitt ([39]) so eloquently observed, it is impossible to make sense of arithmetic without actually using algebraic thinking. To be able to perform multi-digit addition, subtraction, multiplication, and division requires an internalised generality, which cannot easily be articulated, and if such an articulation were available, it would certainly be too complex to be able to follow. Evidence for this goes back 4000 years to Babylonian tablets and Egyptian papyri, in which, rather than attempt to articulate the general, learners were given a 'worked example' and then enjoined to "do it thus"; "do thou likewise"; and similar expressions (Gillings [40]). Indeed, Caleb Gattegno ([41]) argued that a baby in a cot making sounds could be interpreted as exploring basic algebraic ideas of repetition, commutativity, and associativity.

Mary Boole (Tahta [42]) pointed towards the connection between algebra and generalisation by advising learners to "acknowledge your ignorance ... by denoting the as-yet-unknown by some symbol". One way to introduce algebra is therefore through generalisation: inviting children to express generality whenever possible, and using tracking arithmetic as a device to shift attention from the particular to the general. 


\subsection{Tracking Arithmetic}

Tracking arithmetic has been written about in many places (Mason, Johnston-Wilder and Graham [43]; Mason and Johnston-Wilder [44]). Here is one example:

Task 18: ThoaN

Think of a number; add 3; multiply by 2; subtract 7 ; subtract the number you first thought of; add 1; the result is the number you first thought of!

Start with 7, but do not involve that 7 in any calculation. Be careful to distinguish between the 'chosen number $7^{\prime}$ and the structural number in the action 'subtract $7^{\prime}$ !

$$
\begin{gathered}
7 ; 7+3 ; 2(7+3)=2 \times 7+6 ; 2 \times 7+6-7=2 \times 7-1 ; \\
2 \times 7-1-7=1 \times 7-1 ; 1 \times 7-1+1=7 .
\end{gathered}
$$

Note the distinction between the 'subtract $7^{\prime}$ and the isolated starting number 7 .

Now put a cloud around each of the occurrences of the starting number 7 , and then erase the 7 .

The result is a proof that, no matter what number was thought of to begin with-that will always be the result as well.

In brief, tracking arithmetic means having arranged for participants to carry out a sequence of actions, adopt the device of recording, but not actually doing the calculations, so that a single 'chosen' number can be treated as a parameter, and later, replaced by a cloud (standing for "the number that someone is thinking of but hasn't told us"). Subsequently the cloud can be exchanged for a letter when cloud drawing becomes too tedious.

Notice that the key pedagogical action was to substitute (to exchange) each occurrence of the starting number with a cloud or other symbol for an as-yet-unknown number. Mathematicians call this treatment of the starting 7 as a parameter, and it is an instance of Mary Boole's excellent advice. The process of generalising is often a matter of making an exchange of a particular for a symbol, which makes it easier to track the effects on that particular number. Here the exchange of the 'tracked' 7 with a cloud frees the mind from being overwhelmed by arithmetic involving 7 , liberating it to be some as-yet-unknown number, perhaps that someone is thinking of. There is a significant yet apparently trivial shift from 'as-yet-unspecified' number, to 'any' number, and to 'all possible' numbers. This exchange between the symbol as a specific number and the symbol as all possible numbers is the core of generalisation, and the lifeblood of mathematics. Not to experience this repeatedly in school is, to my mind, to be excluded from an essential component of human culture.

ThoaN is an ideal context for intriguing learners, as it takes a while for them to realise that what is essentially going on is undoing a sequence of doings, but not simply by retreating along the same path. Once, when I was using ThoaN with two children to keep them occupied on a long journey, one asked to 'try one'. After a few actions, she soon realised that she had no idea what to do next to recover the number first thought of. Sometime later, after more examples, trials, and discussion, she suddenly said "I have one: ThoaN (pause) that's the number you thought of" followed by giggles all round. It seemed to me to capture the essence of the idea perfectly!

\subsection{Exchange Underpins Substitution}

Whenever a substitution is performed, whether starting from an algebraic statement and substituting a specific value for one or more letters, or making a transformation, the act of substitution is really exchanging one symbol or collection of symbols for another. Learners who have only a tenuous comprehension of the notation $f(x)$ often find expressions such as $f(x+a)-f(a)$ a complete obstacle. Yet all that is being indicated is to exchange the $x$ in $f(x)$, where ever it appears, with the expression $x+a$, and then to subtract from that the result of exchanging $a$ for $x$. A diagram is of course likely to be very helpful ... 
but who draws the diagram? A diagram drawn for me has to be interpreted by me, and then appreciated as germane to current thinking; a diagram drawn by me supports and amplifies thinking, perhaps revealing a gap, or suggesting further construction.

Substitution occurs whenever a general result is used as a formula. For example:

Task 19: area as summation

The area of a trapezium with parallel edges $a$ and $b$ and height $h$ is $\frac{h}{2}(a+b)$.

What is the area of a trapezium with parallel edges 3 and 6 and height 4 ?

What is the area of a trapezium whose edge lengths are $s$ and $s+2$ and height $s$ ?

What is the area of a triangle as suggested by this formula?

What is the sum of $n$ terms of an arithmetic progression whose first and last terms are $a$ and $b$ ?

The connection between the area of a trapezium and an arithmetic progression is enhanced when diagrams are drawn (continuous and discrete). The formula is further enriched when all three displays of the formula $\left(\frac{h(a+b)}{2}, \frac{h}{2}(a+b)\right.$ and $\left.h \frac{a+b}{2}\right)$ are depicted.

Using a formula is an elemental form of exchange or substitution. When treated algebraically, the notion of substitution founded on a rich experience of exchange is much less likely to cause obstruction than when substitution is seen as simply replacing letters by numbers (or vice-versa).

Figure 1 shows the first two in a sequence of shapes made from regular hexagons.

Task 20: edge and hexagon count

How many hexagons will be used to make the nth shape? How many internal and external edges will the shape have? How many external edges?

How many hexagons will be used to make the $3 n+2 n d$ shape?

If there are $3 \mathrm{~h}$ hexagons in one of the shapes, how many internal edges will there be?

How many hexagons will be used to make the shape which has $20+8$ n external edges
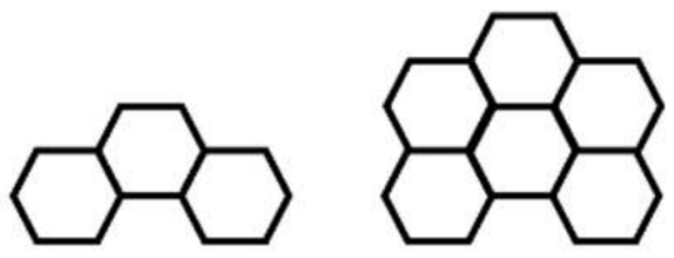

Figure 1. Two stages of a hexagon sequence.

Here, the novice has to keep control of both what they want and what they know, and to use them appropriately (Mason, Burton and Stacey [13]). The more challenging task turns out, as is often the case, to be the undoing version, predicting the number of hexagons from the number of edges. Characterising the form of the possible number of edges often accompanies an undoing task.

\subsection{Substitution as Part of a Construction Leading to Fractals}

Fibonacci and Lucas sequences build subsequent terms from previous terms, so at their core they involve isolating and articulating the construction action, and then repeatedly enacting that action on the previous result, a form of substitution or exchange. The next task has the same form but in a less familiar context.

Articulate for yourself how to draw the $n$th shape in a sequence whose first four terms are displayed in Figure 2. 

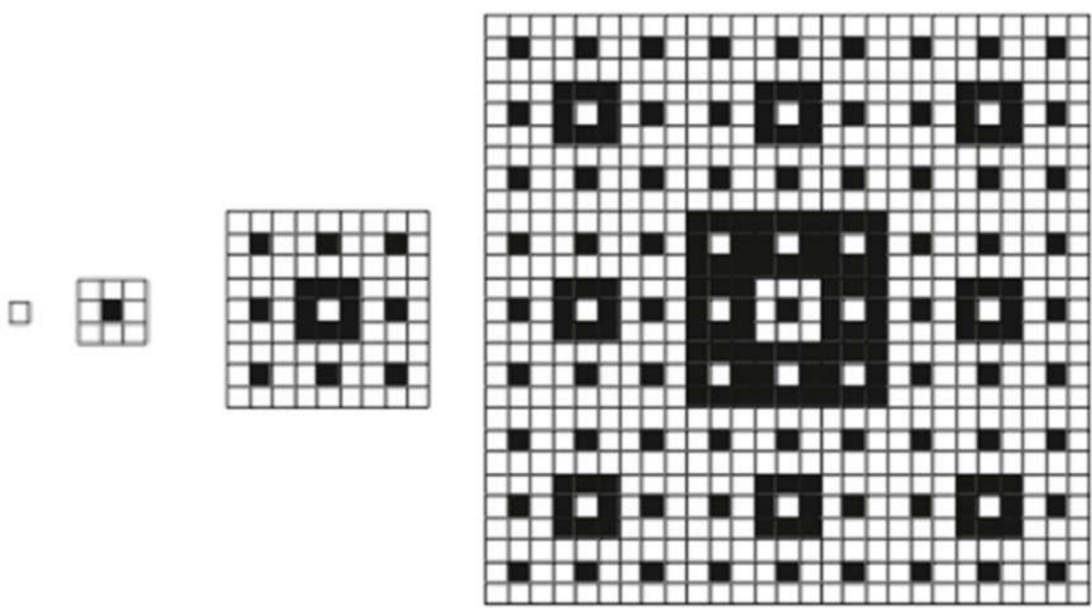

Figure 2. Gaskets.

Task 21: gaskets

At each stage, how many cells in total will there be? How many black? What fraction of the whole is coloured black at each stage? What happens to the density of blacks in the limit?

Notice how the notion of exchange or substitution becomes integral to the specification of subsequent diagrams. This may be reflected in algebraic expressions. The next task provides an even more complicated version of the same idea.

Take a long strip of paper. Fold it end to end by placing the right hand end on top of the left hand end. Make a crease. Repeat this action a number of times. Figure 3 shows the first and second folds, opened to show peaks and valleys.

Task 22: folded strip

Open the strip up and place it on a table so that there is a sequence of peaks and valleys.

Predict the number of peaks, the number of valleys, and the peak-valley sequence after a number of folds.

Denote by $\mathrm{R}$ the folding action (right hand end on top of left hand end) and by $\mathrm{L}$ the opposite (left hand end on top of right hand end).

Write down a 'word' W consisting of a sequence of Rs and Ls. Use this to determine the sequence of folds, reading from right to left (as if the $\mathrm{R}$ and $\mathrm{L}$ were functions being composed). From the word W, predict the number of peaks, the number of valleys and the peak-valley sequence. Which words will give the same sequence of peaks and valleys (or valleys and peaks)?
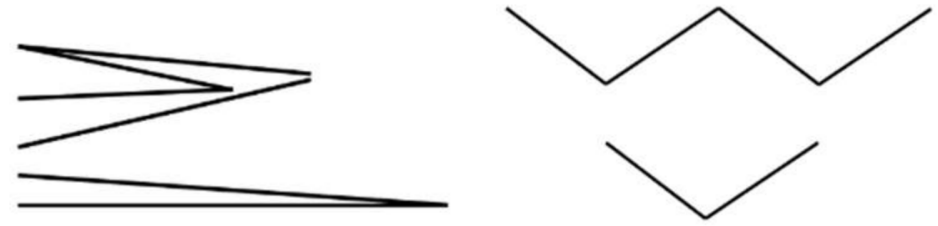

Figure 3. First two folds and corresponding peaks and valleys.

Finding a way of thinking about the effect of the next fold on all the previous folds offers one approach. Another is to look for copies of the previous peak-valley sequence in the new fold, to make a substitution or exchange.

John Stuart Mill declared that arithmetic arises from experience. In this, he was roundly attacked by logicians and formalists. However his idea concerning pebbles (Mill [28]) Chapter VI) was later expanded a little by David Bloor ([44]), which then inspired the following: 
A rectangular array of pebbles is acted upon by sliding the top row to the right so that the pebbles form successive columns, as indicated in Figure 4.

Task 23: pebble arithmetic

Each time a rectangle is formed, the pebbles change colour briefly, to indicate a pause. The number of rows slid since the last rectangle is recorded to form a slide-sequence. In the case here, the slide sequence was 1,1,1 (the last slide is not depicted in the sequence above). That is the doing. The undoing task asks which sequences of numbers can be the slide-sequence for some rectangle of pebbles, and is it possible then to ask, how many pebbles were involved?

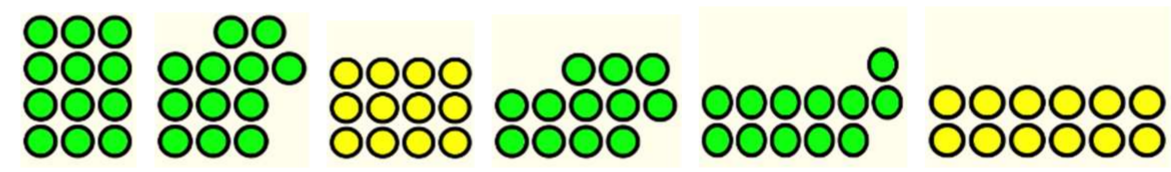

Figure 4. Frames from an animation.

The pedagogic point here is to emphasise the power of formulating and considering 'undoing' or inverse problems. Sometimes there are many solutions, sometimes none, and in the process, learners are exposed to the powerful mathematical theme of organising and characterising, which itself includes considering how many different ways the same result might be achieved, an instance of invariance in the midst of change, and illustrates how mathematics is much more than a set of procedures for answering stylised questions.

\section{Other Examples of Exchange}

\subsection{Statistical}

The root meaning of statistic is as a summary that can stand for (be exchanged with) a mass of data. Mathematically, mean, median, and mode emerge as useful statistics. What is often overlooked is that any measure requires an error bound to indicate the precision of that measure, so a measured length of $1 \mathrm{~m} \pm 0.01$ is measured to the nearest centimetre. This measure, including its error, can be exchanged, or used for, the length in question, giving rise to error arithmetic. In the case of statistics, standard deviation plays the role of error estimate.

In pebble arithmetic, the slide-sequence is a statistic, an indicator or measure of certain aspects of the animation.

\subsection{Multiple Masses}

Isaac Newton came up with the observation that, for the purpose of calculating the effect of a collection of masses (perhaps distributed along a beam as in a beam-balance; perhaps distributed on a thin sheet or joined firmly in some way), it is sufficient to consider the total mass as a single mass acting at a single point (the centre of mass). Thus, a single mass can be substituted or exchanged for a collection of masses, making calculations much simpler.

The notion of reduced mass is also used when considering interactions, in order to describe the motion of one mass in terms of, or relative to, a second mass. This is the parallel or harmonic sum of the masses: $\frac{1}{\frac{1}{m_{1}}+\frac{1}{m_{2}}}=\frac{m_{1} m_{2}}{m_{1}+m_{2}}$.

\section{Conclusions}

Carrying out these tasks for yourself is an important act of preparation for using tasks like them, based on the ideas, or reformulated in some other fashion, because there is a didactic transposition between your experience and what you offer others, inspired or informed by that experience. For example, it is unhelpful to try to 'give learners your experience', because their backgrounds and situations will be different from yours when 
reading or working through your exposition. However, in my experience, effective (and efficient) teaching requires the teacher to have clear objects of learning of content (Marton [34]), to be alert to the presence of core mathematical themes and actions, and to have suitable pedagogic actions available to be enacted. This includes explicit awareness of potential dimensions of possible variation and associated ranges of permissible change. Therefore, a lesson plan consists not of what will be said or done by teachers and learners, but reminders of particular themes and actions that might be useful to invoke, and dimensions to be varied as deemed required. There is no real value in trying to list all possible instances of exchange, or doing and undoing in a given curriculum. Each teacher has to do the work themselves of enriching their sensitivity to notice underpinning themes and core awarenesses, so as to enable fresh actions to be available to be enacted if considered desirable in any given situation.

Furthermore, in prompting and supporting the construction of personal narratives, what matters is not your own narrative, but the impetus to promote sense-making and personal narratives. This requires listening to what learners say, and watching what learners do, rather than listening for what you want to hear, and looking for what you expect or want to see (Davis [45]).

Funding: This research received no external funding.

Institutional Review Board Statement: Not applicable.

Informed Consent Statement: Not applicable.

Data Availability Statement: Not applicable.

Conflicts of Interest: The author declares no conflict of interest.

\section{References}

1. Gardiner, A. Discovering Mathematics: The Art of Investigation; Oxford Science Publications, Clarendon Press: Oxford, UK, 1987.

2. Gardiner, A. Recurring Themes in School Mathematics: Part 1 direct and inverse operations. Math. School 1992, 21, 5-7.

3. Mason, J. Learning $\mathcal{E}$ Doing Mathematics, 2nd ed.; QED Books: York, UK, 1988.

4. SMP 11-16. Doing and Undoing; Cambridge University Press: Cambridge, UK, 1984.

5. Mason, J. Bartering Problems in Arithmetic Books 1450-1890. Br. Soc. Hist. Math. Bull. 1984, 22, 160-181. [CrossRef]

6. Chevallard, Y. La Transposition Didactique; La Pensée Sauvage: Grenoble, France, 1985.

7. Bosch, M.; Gascon, J. Twenty-Five Years of the Didactic Transposition. ICMI Bull. 2006, 58, 51-65.

8. Simon, M.; Tzur, R. Explicating the Role of Mathematical Tasks in Conceptual Learning: An elaboration of the hypothetical learning trajectory. Math. Think. Learn. 2004, 6, 91-104. [CrossRef]

9. Deleuze, G.; Guattari, F. A Thousand Plateaus: Capitalism and Schizophrenia; Bloomsbury: London, UK, 1980.

10. Vergnaud, G. The nature of mathematical concepts. In Learning and Teaching Mathematics: An International Perspective; Nunes, T., Bryant, P., Eds.; Psychology Press: London, UK, 1997; pp. 5-8.

11. Mason, J.; Johnston-Wilder, S. Designing and Using Mathematical Tasks; republished 2006; Open University: Milton Keynes, UK; Tarquin: St. Albans, UK, 2004.

12. Brousseau, G. The Crucial Role of the Didactical Contract in the Analysis and Construction of Situations in Teaching and Learning mathematics. In Theory of Mathematics Education; Paper 54; Steiner, H., Ed.; Institut fur Didaktik der Mathematik der Universitat: Bielefeld, Germany, 1984; pp. 110-119.

13. Mason, J.; Burton, L.; Stacey, K. Thinking Mathematically (Second Extended Edition); Prentice Hall (Pearson): Harlow UK, 1982.

14. Cuoco, A.; Goldenberg, P.; Mark, J. Habits of Mind: An organizing principle for mathematics curricula. J. Math. Behav. 1996, 15, 375-402. [CrossRef]

15. Schoenfeld, A. Mathematical Problem Solving; Academic Press: New York, NY, USA, 1985.

16. Mason, J. Doing \& Undoing, Project Update; Open University: Milton Keynes, UK, 1988.

17. Mason, J. Learning and Doing Mathematics; MacMillan: London, UK, 1988.

18. Mason, J.; Johnston-Wilder, S. Fundamental Constructs in Mathematics Education; RoutledgeFalmer: London, UK, 2004.

19. Gattegno, C. The Science of Education Part I: Theoretical considerations; Educational Solutions: New York, NY, USA, 1987.

20. Mason, J. Only Awareness is Educable. In Teaching, Learning and Mathematics; Bloomfield, A., Harries, T., Eds.; Association of Teachers of Mathematics: Derby, UK, 1994; pp. 28-29.

21. Mason, J. Educating Awareness through Mediated Action. In On Teaching and Learning Mathematics with Awareness; Hewitt, D., Coles, A., Ingram, J., Eds.; Association of Teachers of Mathematics: Derby, UK, 2018; pp. 71-76. 
22. Mason, J. Enabling Teachers to be Real Teachers: Necessary levels of awareness and structure of attention. J. Math. Teach. Educ. 1998, 1, 243-267. [CrossRef]

23. Mason, J. On The Structure of Attention in the Learning of Mathematics. Aust. Assoc. Math. Teach. 2003, 59, 17-25.

24. Watson, A.; Mason, J. Mathematics As a Constructive Activity: Learners Generating Examples; Erlbaum: Mahwah, NJ, USA, 2005.

25. Bruner, J. The Narrative Construction of Reality. In Rappresentazioni e Narrazioni; Ammanti, M., Stern, D., Eds.; Laterze: Roma-Bari, Italy, 1991; pp. 17-42.

26. Chi, M.; Bassok, M. Learning from examples via self-explanation. In Knowing, Learning and Instruction: Essays in Honour of Robert Glaser; Resnick, L., Ed.; Erlbaum: Hillsdale, MI, USA, 1989.

27. Hamilton, E.; Cairns, H. (Eds.) Plato: The Collected Dialogues Including the Letters; Phaedrus. Bollingen Series LXXI; Princeton University Press: Princeton, NJ, USA, 1961.

28. Mill, J.S. A System of Logic, Ratiocinative and Inductive, Being a Connected View of the Principles of Evidence, and the Methods of Scientific Investigation, 8th ed.; Harper \& Bros: New York, NY, USA, 1882.

29. Montessori, M. The Montessori Method; Transaction: London, UK, 1964.

30. NRich. Available online: https:/ / nrich.maths.org/10059 (accessed on 14 February 2021).

31. BBC. Available online: https://www.bbc.co.uk/cbeebies/topics/numeracy (accessed on 14 February 2021).

32. Gripton, C.; Pawluch, D. Counting Collections in the Early Years. Math. Teach. 2021, 275, 6-10.

33. Marton, F.; Booth, S. Learning and Awareness; Lawrence Erlbaum: Hillsdale, MI, USA, 1997.

34. Marton, F. Necessary Conditions for Learning; Routledge: Abingdon, UK, 2015.

35. Barton, B.; Fairhall, U.; Trinick, T. Tikanga Reo Tatai: Issues in the development of a Maori Mathematics register. Learn. Math. 1998, 18, 3-9.

36. Borden, L. The 'Verbification' of Mathematics: Using the grammatical structures of mi'kmaq to support student learning. Learn. Math. 2011, 31, 8-13.

37. James, N.; Mason, J. Towards Recording. Visible Lang. 1982, 16, 249-258.

38. Thomsen, G. The Treatment of Elementary Geometry by a Group-Calculus. Math Gazette. 1933, 17, 230-242. [CrossRef]

39. Hewitt, D. Approaching Arithmetic Algebraically. Math. Teach. 1998, 163, 19-29.

40. Gillings, R. Mathematics in the Time of the Pharaohs; Dover: New York, NY, USA, 1972; Reprinted in 1982.

41. Gattegno, C. What We Owe Children: The Subordination of Teaching to Learning; Routledge \& Kegan Paul: London, UK, 1970.

42. Tahta, D. A Boolean Anthology: Selected Writings of Mary Boole on Mathematics Education; Association of Teachers of Mathematics: Derby, UK, 1972.

43. Mason, J.; Johnston-Wilder, S.; Graham, A. Developing Thinking in Algebra; Sage (Paul Chapman): London, UK, 2005.

44. Bloor, D. Knowledge E Social Imagery; University of Chicago Press: Chicago, IL, USA, 1976.

45. Davis, B. Teaching Mathematics: Towards a Sound Alternative; Ablex: New York, NY, USA, 1996. 